\title{
¿De que se mueren los recién nacidos en la Argentina?
}

¿De que se mueren los recién nacidos en Argentina?. Lomuto Celia. Revista Mat Inf Ramón Sardá 1998; XVII: N 1, 31-35.

\section{Objetivo}

Describir y analizar las causas de muertes neonatales (de 0 a 28 días) ocurridas en la República Argentina, durante 1995.

\section{Diseño}

Estudio descriptivo.

\section{Lugar}

República Argentina.

\section{Participantes}

Total de muertes entre 0 y 28 días de vida, obtenidas a partir de fuentes de datos secundarias, Estadísticas Vitales de 1995 publicadas por el Ministerio de Salud y Acción Social.

\section{Metodología}

Para analizar las causas de muerte se utilizó la Clasificación Internacional de Enfermedades (CIE-9, 1975). Se agruparon las causas de muerte en los siguientes grupos: 1) perinatales, malformaciones congénitas, infecciones, causas varias (tumores, enfermedad del aparato digestivo, del SNC, cardiovascular, respiratorio, etc.), accidentes y homicidios, mal definida y diagnósticos incoherentes; 2) causas de muerte que potencialmente podrían reducirse (criterio de reducibilidad) y 3) según peso al nacer.

\section{Medición de resultados principales}

Del total de fallecidos en menores de un año, el número y porcentaje de muertes que correspondieron al período neonatal (precoz -0 a 6 días- y tardío -7 a 27 días-).

\section{Resultados principales}

En el año 1995 nacieron vivos 658.735 niños, y fallecieron 14.606 menores de un año, de los cuales 8.942 lo hicieron en el período neonatal (61\%).

Las principales causas de muerte neonatal son las perinatales $72 \%$ y las anomalías congénitas $18 \%$, contribuyendo las restantes en menor proporción. Entre las perinatales se destacan: hipoxia, asfixia y otras afecciones del feto o del recién nacido que suman el 31,9 $\%$; el crecimiento fetal lento, la desnutrición o la inmadurez fetal alcanzan el $20,8 \%$; y otras afecciones del período perinatal el 15 $\%$. Según el criterio de reducibilidad se observó que el $61,6 \%$ de todas las muertes son reducibles y entre ellas el $31,4 \%$ en el embarazo. El 59,1\% de las muertes entre 0 y 27 días correspondieron a niños cuyo peso al nacer fue menor a 2.500 gramos.

\section{Conclusiones}

El $61,6 \%$ de todas las muertes neonatales son potencialmente reducibles y entre ellas el $31,4 \%$ controlables durante el embarazo.

\section{COMENTARIO}

En principio, es necesario tener presente la influencia de la calidad de los datos en el valor del indicador, en este caso mortalidad neonatal. Esto se debe a que, además del posible subregistro de las muertes y/o de las causas, podrían sumarse los inconvenientes y las dificultades del médico certificador ${ }^{1}$.

Los resultados de este estudio muestran que en la mortalidad neonatal prevalecen factores relacionados con la atención de la salud y las condiciones congénitas. Los primeros, que incluyen salud de la madre, control del embarazo, atención del parto y del niño durante los primeros días de vida; son responsables de casi dos tercios de las muertes en esta edad.

De los neonatos fallecidos, el 59,1\% pesó por debajo de 2.500 gramos y el $37,6 \%$ pesó menos de 1.500 gramos. Esta situación, de alta incidencia en países o regiones subdesarrolladas, se acompaña frecuentemente de otros indicadores de riesgo elevado, como edad materna, paridad, edad gestacional, nivel de instrucción materno, entre otros ${ }^{2-3-4}$. La mejoría en la atención de la salud puede disminuir la morbimortalidad y las secuelas de este grupo de alto riesgo, pero no su incidencia, ya que ésta es producto del defecto de numerosas variables que dependen del desarrollo social y cuya erradica- ción necesita de medidas estructurales, capaces de producir impacto pero a muy largo plazo.

El análisis de las causas de muerte según Criterios de Reducibilidad, comenzó a utilizarse en Argentina a partir de $1985^{5}$. Esto permitió en función del riesgo, orientar las acciones en el área materno infantil, guiar la normatización de patologías prevalentes en el medio y sustentar la planificación y programación de actividades. La elevada incidencia de muertes reducibles, casi el $62 \%$; enfatiza la inadecuada calidad del sistema de salud y la necesidad de priorizar las acciones en el control prenatal.

Desde el punto de vista de la prevención de la mortalidad neonatal, es importante cortar la cadena de acontecimientos a través de: diagnóstico precoz del embarazo; adecuado control prenatal, del trabajo de parto y parto; correcta recepción y reanimación del recién nacido e instauración de tratamientos específicos.

El objetivo primordial es prevenir que opere la causa precipitante; siendo las estadísticas de causas de muerte una de las herramientas más útiles para el conocimiento de los problemas de salud de una población, para sustentar la toma de decisiones y orientar los programas tendientes a facilitar la instrumentación de medidas correctivas.

\section{Dra. Nanci Giraudo}

Unidad de Medicina Familiar y Preventiva. Hospital Italiano de Buenos Aires.

\footnotetext{
Referencias

1. Segura A, Gispert R. El médico y la certificación de las causas de defunción. Med Clin (Barc) 1987,89:415 - 422

2. Puffer R, Serrano C. El peso al nacer, la edad materna y el orden de nacimiento. Tres importantes determinantes de la mortalidad infantil. OPS/OMS - Publ. Cient. 724,1975; 1-46

3. Ministerio de Salud y Acción Social - Secretaria de Salud - Programa nacional de Estadísticas de Salud. "Situación sanitaria de los menores de 1" año." Serie 10, N * 5: 1992. Buenos Aires, Argentina.

4. Wilcox A, Akjaervan R, Buekens Py cols. Birth weight and perinatal mortality. A comparison of the United States and Norway. JAMA 1995, $273: 709$ - 711.

5. Ministerio de Salud y Acción Social - Secretaria de Salud - Programa nacional de Estadísticas de Salud. "Agrupamiento de causas de muerte de menores de un año basado en la aplicación de criterios de evitabilidad, 1979 - 1980 - 1981". Boletín N ${ }^{\circ}$ 50: 1985. Buenos Aires, Argentina.
} 